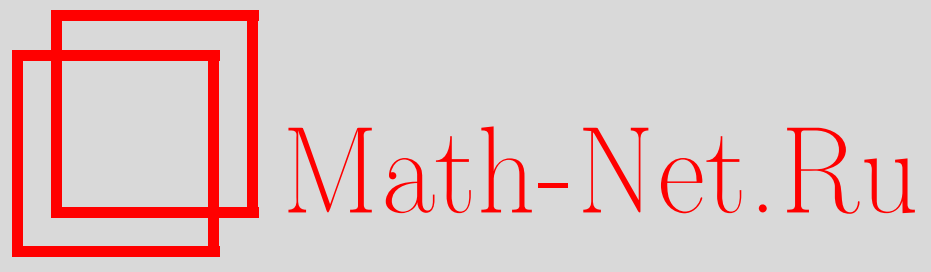

А. О. Ремизов, О геодезических в метриках с особенностями типа Клейна, УМH, 2010, том 65, выпуск 1, 187188

DOI: https://doi.org/10.4213/rm9329

Использование Общероссийского математического портала Math-Net.Ru подразумевает, что вы прочитали и согласны с пользовательским соглашением http://www . mathnet.ru/rus/agreement

Параметры загрузки:

IP : 34.227 .88 .159

26 апреля 2023 г., 13:02:53

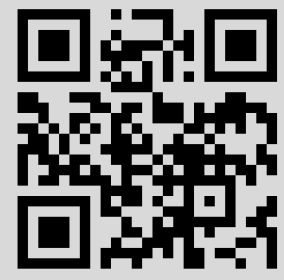




\section{О геодезических в метриках с особенностями типа Клейна}

\section{А. О. Ремизов}

В этой заметке анонсируются некоторые результаты о геодезических в метрике

$$
d s^{2}=\frac{\alpha(t, x)\left(d x^{2}+\varepsilon d t^{2}\right)}{\omega^{r}(t, x)}, \quad \varepsilon= \pm 1,
$$

где $\alpha(t, x)>0$ и $\omega(t, x)$ - гладкие (класса $C^{\infty}$ ) функции, $r>0$ - вещественное число. Предполагается, что уравнение $\omega(t, x)=0$ задает гладкую регулярную кривую $A$, т. е. во всех точках $(t, x) \in A$ выполнено условие $\nabla \omega \neq 0$. В случае $\varepsilon=-1$ предполагается также, что кривая $A$ не касается радикала формы $d x^{2}+\varepsilon d t^{2}$, т. е. во всех точках $(t, x) \in A$ выполнено условие $\left|\omega_{t}^{\prime}\right| \neq\left|\omega_{x}^{\prime}\right|$. Цель работы - исследование особенностей геодезического потока и геодезических, проходящих через точки кривой $A$, вблизи этих точек. Все результаты локальные и с помощью изотермических координат переносятся на метрики вида $d s^{2}=Q / \omega^{r}$, где $Q$ - произвольная гладкая невырожденная метрика (если $Q$ индефинитна, то требуется, чтобы кривая $A=\{\omega=0\}$ не касалась $\operatorname{Rad} Q)$.

Метрики вида (1) суть обобщение метрики Клейна, используемой в модели плоскости Лобачевского $(r=2)$. Они также связаны с метрикой Клеро-Луивилля $(r=1)$, имеющей приложения в теории управления. Так, в [1] рассматривается метрика вида

$$
d s^{2}=d x^{2}+\frac{g\left(x^{2}, t\right)}{x^{2}} d t^{2}=\frac{x^{2} d x^{2}+g\left(x^{2}, t\right) d t^{2}}{x^{2}},
$$

где $g$ - положительная гладкая функция $(t$ и $x$ - угловые координаты на сфере, линия $A=\{x=0\}$ - экватор). В случае, если $g\left(x^{2}, t\right) \equiv 1$, это - хорошо известная метрика Грушина [2], [3]. Заменой $\tilde{x}=x^{2}$ метрика (2) приводится к виду $d s^{2}=Q /(4 \tilde{x})$ с квадратичной формой $Q=d \tilde{x}^{2}+4 g(\tilde{x}, t) d t^{2}$, а последняя с помощью изотермических координат приводится к виду (1) с $r=1$ и $\varepsilon=+1$.

Геодезические в метрике (1) суть экстремали уравнения Эйлера-Лагранжа для функционала длины с $L^{2}=\alpha\left(p^{2}+\varepsilon\right) / \omega^{r}$, где $p=d x / d t$. Преобразуя, получаем уравнение

$$
2 \varepsilon \alpha \omega \frac{d p}{d t}=\left(\mu_{1} p-\varepsilon \mu_{0}\right)\left(p^{2}+\varepsilon\right), \quad p=\frac{d x}{d t},
$$

где $\mu_{1}=r \alpha \omega_{t}^{\prime}-\alpha_{t}^{\prime} \omega$ и $\mu_{0}=r \alpha \omega_{x}^{\prime}-\alpha_{x}^{\prime} \omega$. В пространстве $(t, x, p)$ уравнение (3) задает поле направлений

$$
\dot{t}=2 \varepsilon \alpha \omega, \quad \dot{x}=2 p \varepsilon \alpha \omega, \quad \dot{p}=\left(\mu_{1} p-\varepsilon \mu_{0}\right)\left(p^{2}+\varepsilon\right),
$$

проекции интегральных кривых которого на плоскость $(t, x)$ вдоль оси $p$ являются геодезическими. Поле (4) обладает двумя важными свойствами.

Во-первых, для каждой точки $q=(t, x) \in A$ и каждого $p$ такого, что $\left(\mu_{1} p-\varepsilon \mu_{0}\right) \times$ $\left(p^{2}+\varepsilon\right) \neq 0$, поле (4) имеет ровно одну интегральную кривую, проходящую через точку $(q, p)$, проекция которой на плоскость $(t, x)$ дает точку $q$ и не соответствует никакой геодезической (это - прямая в пространстве $(t, x, p)$, параллельная оси $p$ ). Таким образом, геодезические, проходящие через точку $q \in A$, являются проекциями интегральных кривых поля (4), проходящих через его особые точки $(q, p)$, т. е. $\left(\mu_{1} p-\varepsilon \mu_{0}\right)\left(p^{2}+\varepsilon\right)=0$.

Во-вторых, особые точки поля (4) определяются уравнениями $\omega=0$ и $\left(\mu_{1} p-\varepsilon \mu_{0}\right) \times$ $\left(p^{2}+\varepsilon\right)=0$, задающими в случае $\varepsilon=+1$ кривую $W_{0}^{c}=\left\{q \in A, p=\varepsilon \mu_{0} / \mu_{1}\right\}$. В случае $\varepsilon=-1$ к ней добавляется еще пара кривых $W_{ \pm}^{c}=\{q \in A, p= \pm 1\}$. В силу условия $\left|\omega_{t}^{\prime}\right| \neq\left|\omega_{x}^{\prime}\right|$ кривые $W_{0}^{c}, W_{ \pm}^{c}$ вблизи $A$ не пересекаются и являются центральными 
многообразиями поля $\left(W^{c}=W_{0}^{c} \cup W_{+}^{c} \cup W_{-}^{c}\right)$. Спектр линейной части поля направлений (4) определен с точностью до пропорциональности и в точках кривой $W_{0}^{c}$ равен $(2, r, 0)$, а в точках кривых $W_{ \pm}^{c}$ равен $(1,-r, 0)$. При этом собственному значению $r$ или $-r$ соответствует собственное направление $\partial_{p}$.

Комбинируя результаты работ [4], [5], мы получаем гладкие орбитальные нормальные формы ростка поля (4) в его особых точках. Так, в любой точке $(p, q) \in W_{0}^{c}$ росток (4) имеет нормальную форму

$$
\begin{array}{llll}
\dot{\xi}=r \xi, & \dot{\eta}=2 \eta, & \dot{\zeta}=0, & \text { если } \max \{r / 2,2 / r\} \notin \mathbb{N}, \\
\dot{\xi}=n \xi+\varphi(\zeta) \eta^{n}, & \dot{\eta}=\eta, & \dot{\zeta}=0, & \text { если } \max \{r / 2,2 / r\}=n \in \mathbb{N} .
\end{array}
$$

Если $\varphi(0) \neq 0$, то коэффициент $\varphi(\zeta)$ в нормальной форме (6) может быть заменен на 1. Если $\varphi(\zeta)$ имеет в начале координат нуль конечного порядка $s$, то $\varphi(\zeta)$ может быть заменен на $\zeta^{s}$. В случае $r=1$ мы всегда имеем нормальную форму (6) с $n=2$ и $\varphi(\zeta) \equiv 0$. В случае $r=2$ тождество $\varphi(\zeta) \equiv 0$ имеет место, например, если функция $\omega(t, x)$ линейна и ограничение $\alpha(t, x)$ на прямую $A$ тождественно равно константе.

В любой точке $(p, q) \in W_{ \pm}^{c}$ росток (4) имеет нормальную форму

$$
\begin{array}{llll}
\dot{\xi}=\xi, & \dot{\eta}=-r \eta, & \dot{\zeta}=0, & \text { если } r \notin \mathbb{Q}, \\
\dot{\xi}=n \xi, & \dot{\eta}=-m \eta \Phi(\rho, \zeta), & \dot{\zeta}=\rho \Psi(\rho, \zeta), & \text { если } r \in \mathbb{Q},
\end{array}
$$

где $\rho=\xi^{m} \eta^{n}$ - резонансный моном, $r=m / n, m, n \in \mathbb{N},(m, n)=1$ и $\Phi(0,0)=1$. Если $\Psi(0,0) \neq 0$, то $\Phi(\rho, \zeta)$ и $\Psi(\rho, \zeta)$ в $(8)$ могут быть заменены на 1 . Условие $\Psi(0,0) \neq 0$ выполнено для метрики (1) общего положения, при $r=1$ оно выполнено вообще всегда. Топологически обе нормальные формы (7) и (8) эквивалентны ростку $\dot{\xi}=\xi$, $\dot{\eta}=-\eta, \dot{\zeta}=0$.

Чтобы получить портрет геодезических, выходящих из точки $q \in A$ с касательным направлением $p_{0}=\varepsilon \mu_{0} / \mu_{1}$, нужно спроектировать на плоскость $(t, x)$ вдоль оси $p$ интегральные кривые поля (4), лежащие на инвариантном слое $(\zeta=c$ (const) в нормальной форме (5) или (6)), проходящем через данную точку $\left(q, p_{0}\right)$. Это дает пучок геодезических $\gamma_{k}, k \in \mathbb{R}$, с общей 1-струей в точке $q$. Струи высших порядков зависят от $r$ и $\varphi(c)$. Если $r<2$, то все $\gamma_{k}$, кроме одной, имеют в точке $q$ бесконечную вторую производную (при $r=1$ это полукубические параболы с вершиной в $q$ ).

Пусть теперь $r=2 n, n \in \mathbb{N}$, и мы имеем нормальную форму (6). Если $\varphi(c) \neq 0$, то все $\gamma_{k}$ - класса $C^{n}$ с общей $n$-струей и бесконечной производной порядка $n+1$. Если $\varphi(c)=0$, то все $\gamma_{k}-$ класса $C^{\infty}$ с общей $n$-струей и разными $(n+1)$-струями. ${ }^{1} \mathrm{~B}$ случае $\varepsilon=+1$ все геодезические, выходящие из точки $q=\left(t_{*}, x_{*}\right) \in A$, исчерпываются пучком $\gamma_{k}, k \in \mathbb{R}$. В случае $\varepsilon=-1$ к ним добавляются две изотропные линии $\gamma_{ \pm}$: $\left(x-x_{*}\right)= \pm\left(t-t_{*}\right)$ с касательными направлениями $p= \pm 1$.

\section{Список литературы}

[1] B. Bonnard, J.-B. Caillau, E. Trélat, Dynamical Systems and Differential Equations. Proceedings of the 6th AIMS International Conference, Discrete Contin. Dyn. Syst., 2007, 145-154. [2] В. В. Грушин, Матем. сб., 84:2 (1971), 163-195. [3] Р. Р. Файзуллин, Сиб. матем. журн., 44:6 (2003), 1377-1384. [4] R. Roussarie, Astérisque, 30, Société Mathématique de France, Paris, 1975, 1-182. [5] А. О. Ремизов, СМФН, 19 (2006), 131-170.

\section{А. О. Ремизов (А. O. Remizov)}

International School for Advanced Studies (SISSA)

E-mail: alexey-remizov@yandex.ru, remizov@sissa.it
Представлено М. И. Зеликиным Принято редколлегией 11.11.2009

\footnotetext{
${ }^{1}$ Например, для обычной метрики Клейна (1) с $r=2, \varepsilon=+1$ и $\alpha(t, x)=1, \omega(t, x)=t$ в нормальной форме $(6) \varphi(\zeta) \equiv 0$. Геодезические - окружности $\left(x-x_{0}\right)^{2}+t^{2}=R^{2}$ и прямые $x=c-$ являются $C^{\infty}$-гладкими вблизи точек пересечения с абсолютом $A=\{t=0\}$ и для фиксированной точки $q \in A$ различаются 2-струями.
} 